\title{
Measurement of Indeterminacy in Packings of Perfectly Rigid Disks
}

\author{
Sean McNamara* and Hans Herrmann \\ Institut für Computerphysik, Universität Stuttgart, 70569 Stuttgart, GERMANY
}

(Dated: November 7, 2018)

\begin{abstract}
Static packings of perfectly rigid particles with Coulomb friction are investigated theoretically and numerically. The problem of finding the contact forces in such packings is formulated mathematically. Letting the values of the contact forces define a vector in a high-dimensional space enables us to consider the set of all possible contact forces as a region embedded in this same space. It is found that the boundary of the set is connected with the presence of sliding contacts, suggesting that a stable packing should not have more than $2 M-3 N$ sliding contacts in two dimensions, where $M$ is the number of contacts and $N$ is the number of particles.

These results are used to analyze packings generated in different ways by either molecular dynamics or contact dynamics simulations. The dimension of the set of possible forces and the number of sliding contacts agrees with the theoretical expectations. The indeterminacy of each component of the contact forces are found, as well as an estimate for the diameter of the set of possible contact forces. We also show that contacts with high indeterminacy are located on force chains. The question of whether the simulation methods can represent a packing's memory of its formation is addressed.
\end{abstract}

PACS numbers: $45.70 . \mathrm{Cc}$

\section{INTRODUCTION}

The physics of granular materials involves two very different length scales. The first length scale is associated with the size of the particles. If we wish to give an accurate value of the density, or describe the movement of the particles, it suffices to give the particle positions with an accuracy of some fraction of their radii. We call this length scale the "kinetic" length scale $\ell_{\text {kin }}$, and take it to be of order the particle radius. When two particles touch, inter-particle forces at contacts are generated by tiny deformations that can be characterized by a second length scale, that we will call the "elastic" length scale $\ell_{\mathrm{el}}$.

One normally has $\ell_{\mathrm{el}} \ll \ell_{\text {kin. }}$. If one takes two marbles, $\ell_{\text {kin }}$ is about half a centimeter. But $\ell_{e l}$ is not visible to the naked eye, as one can confirm by pushing the marbles together, and trying to observe their deformation. Because one often has $\ell_{\mathrm{el}} \ll \ell_{\mathrm{kin}}$, it is tempting to derive simplified numerical or theoretical approaches by taking the limit $\ell_{e l} \rightarrow 0$, corresponding to infinitely rigid particles. One example is the inelastic hard sphere model, where collisions are assumed to be instantaneous. Instead of resolving the forces during a collision, one simply calculates the post-collisional velocities as a function of the pre-collisional ones.

The inelastic hard sphere model opens the way to the application of kinetic theory and the use of event driven computer simulations. Both of these techniques have been applied successfully to a wide variety of granular flows [1, 2, 3]. But the neglected length scale $\ell_{\mathrm{el}}$ takes its revenge in an unexpected way. If the collisions are dissipative, "inelastic collapse" can occur: there can be

*Email address sean@ica1.uni-stuttgart.de an infinite number of collisions in finite time [4, 5]. In event driven simulations, it is necessary to modify the simple inelastic hard sphere model to avoid this singularity. There are two general approaches. In the first approach, each particle carries a clock that records the time of its last collision. When two particles collide, one checks their clocks to see if either one has had a collision less than some time $t_{c}$ ago. If so, the collision dissipates no energy, otherwise, the collision proceeds normally. The time $t_{c}$ corresponds to the duration of a collision [6]. Alternatively, one can make the restitution coefficient depend on impact velocity in such a way that the energy dissipation goes to zero as the impact velocity vanishes 7].

Another numerical method based on the approximation $\ell_{\mathrm{el}} \rightarrow 0$ is contact dynamics (hereafter "CD") $[8,9,10]$. In this method, the contact forces are calculated by requiring them to prevent particle interpenetration and to minimize sliding. In this case, the neglected length scale takes its revenge by causing indeterminacy [8]. In most cases, there are many possible force networks that satisfy the constraints that are imposed on them. This raises several questions addressed in this paper: First of all, how big is the set of possible contact forces? Secondly, how are the forces chosen by CD distinguished from all the other possible solutions? Finally, how do the forces chosen by CD differ from those calculated by soft-particle "molecular dynamics" (hereafter "MD") [1], where the particle deformations are explicitly treated? This paper addresses these questions.

Another recently proposed approach similar to CD is the "force network ensemble" [12]. The force network ensemble is the set of all possible force networks that could exist in a given configuration of rigid particles. In Ref. 12], this ensemble was sampled to obtain force distributions, which are compared with MD simulations. Parallels were drawn between the force network ensemble 
and the ensembles of statistical mechanics, so that one could calculate properties of packings by averaging over the force network ensemble. But are all members of the ensemble equally likely to be realized?

These questions have begun to be addressed. For example, it has been shown that the contact forces in static assemblies of frictionless grains can be uniquely determined [13]. In Ref. 14], the CD algorithm was adapted to sample the force network ensemble, allowing the authors to estimate its size. The authors found that the ensemble was not uniformly sampled, and that the force state generated by the dynamics had special properties. They also carried out a detailed study of the influence of tangential friction, and showed, that indeterminacy disappears in the limit of vanishing friction, consistent with Ref. [13].

This paper takes a different, but complementary approach. We investigate the structure of the force network ensemble mathematically, and show (in agreement with 14]) that it is a convex set. In addition, we show that the boundaries of the set are associated with contacts where the Coulomb condition is marginally fulfilled. These findings place an upper limit on the number of such contacts that can exist in a static packing, and a lower limit on the dimension of the force network ensemble. We show how to locate the extremal points, enabling us to calculate the indeterminacy of the contact forces and to estimate the size of the force ensemble network. We also study the difference between the MD and CD calculation methods.

This paper is organized into two main parts. Sec. I presents a mathematical formulation of the problem of finding the contact forces in a packing of infinitely rigid disks with Coulomb friction. It is shown that this problem is equivalent to finding the intersection of a cone and a linear subspace in a high dimensional space. In Sec. III we apply these ideas numerically to static packings of about 100 particles, and answer several questions about the range of possible forces that could exist in the packing, and how they are related to the MD and $\mathrm{CD}$ solutions.

\section{MATHEMATICAL FORMULATION}

\section{A. Definition of the contact matrix}

We study a system of $N$ two-dimensional, circular grains at rest under gravity $g$ in a rectangular container. We label each grain with a unique integer $i, 1 \leq i \leq N$. Particle $i$ is characterized by its mass $m_{i}$, radius $r_{i}$, position $\vec{r}_{i}$, velocity $\vec{v}_{i}$, momentum of inertia $I_{i}$, and angular velocity $\omega_{i}$. The fixed walls of the container could be considered as particles with infinite mass, but it is more convenient to simply leave them out of the analysis.

Let $M$ be the number of contacts between the $N$ grains. Each contact can also be labeled with a unique integer $\alpha, 1 \leq \alpha \leq M$. Contact $\alpha$ is characterized by the two touching grains $i$ and $j$. Given the positions of particles $i$ and $j$, it is possible to define two unit vectors $\hat{n}_{\alpha}$ and $\hat{t}_{\alpha}$ that point in the directions normal to and tangent to the contact, respectively. At the contact, the two particles exert a normal force $R_{\alpha}$ and a tangential force $T_{\alpha}$ on each other.

To calculate the motion of the particles, it is necessary to know the force $\overrightarrow{f_{i}}$ and the torque $\tau_{i}$ on each particle due to the contacts. Since $\vec{f}_{i}$ and $\tau_{i}$ depend linearly on the contact forces, one can write

$$
\mathbf{f}=\mathbf{c F}
$$

Here, the contact forces and the forces experienced by the particles have been collected together into two column vectors $\mathbf{F}$ and $\mathbf{f}$ :

$$
\mathbf{f}=\left(\begin{array}{c}
f_{1 x} \\
f_{1 y} \\
\tau_{1} \\
\vdots \\
f_{N x} \\
f_{N y} \\
\tau_{N}
\end{array}\right), \quad \mathbf{F}=\left(\begin{array}{c}
R_{1} \\
T_{1} \\
\vdots \\
R_{M} \\
T_{M}
\end{array}\right)
$$

Note that $\mathbf{f} \in \mathbb{R}^{3 N}$ and $\mathbf{F} \in \mathbb{R}^{2 M}$. The matrix $\mathbf{c}$ has dimensions $3 N \times 2 M$, and is called the contact matrix. It contains information about which particles touch each other, and the geometry of the contacts. In Sec. IB we give explicit expressions for the components of $\mathbf{c}$. Following [8], we call any particular value of $\mathbf{F}$ a "contact state" because it gives the state of all the contacts in the granular packing.

Using this notation, we can easily write down the system of equations that must be solved in order to find the forces in a static granular packing under gravity. If the particles do not move, the contact forces must balance the gravitational acceleration:

$$
\mathbf{f}=-\mathbf{M g} .
$$

Here, $\mathbf{M} \in \mathbb{R}^{3 N} \times \mathbb{R}^{3 N}$ is a diagonal matrix containing the masses and moments of inertia of the particles:

$$
\mathbf{M}=\left(\begin{array}{ccccccc}
m_{1} & & & & & & \\
& m_{1} & & & & & \\
& & I_{1} & & & & \\
& & & \ddots & & & \\
& & & & m_{N} & & \\
& & & & & m_{N} & \\
& & & & & & I_{N}
\end{array}\right) .
$$

The vector $\mathbf{g}$ contains the gravitational accelerations of all the particles, organized in the same way as $\mathbf{f}$ in Eq. (2).

In contact dynamics, the unknowns are the contact forces $\mathbf{F}$, not the forces on each particle, so we use Eq. (1) to re-write Eq. (3) as

$$
\mathbf{c F}=-\mathbf{M g}
$$


If $\mathbf{c}$ were not singular, one could find a unique solution by inverting Eq. (5):

$$
\mathbf{F}=-\mathbf{c}^{-1} \mathbf{M g}
$$

But if $\mathbf{c}$ is singular, Eq. (5) has no unique solution, because there exist vectors $\mathbf{F}_{0} \neq 0$ such that

$$
\mathbf{c F}_{0}=0 \text {. }
$$

Physically, this means that there are contact states that exert no net force on the particles. This is the source of indeterminacy in granular packings [8]. Once we have found a solution to Eq. (5), we can construct an infinite number of solutions by adding multiples of $\mathbf{F}_{0}$. However, not all of these solutions are possible, for reasons discussed in Sec. IIC

In general, $\mathbf{c}$ is singular, and its null space $\mathbb{C}_{0}$, plays a very important role in this paper. By considering the dimensions of $\mathbf{c}$, one can establish a lower bound on the dimension of $\mathbb{C}_{0}$. c can be applied to any vector in $\mathbb{R}^{2 M}$, so its domain has dimension $2 M$. c maps this vector onto another vector in $\mathbb{R}^{3 N}$, so its range has dimension of at most $3 N$. Since the dimension of the range and null space must add to the dimension of the domain, we have

$$
\operatorname{dim} \mathbb{C}_{0} \geq 2 M-3 N
$$

If $2 M \leq 3 N, \operatorname{dim} \mathbb{C}_{0}$ could vanish, but this corresponds to a coordination number of less than 3 . Therefore, we expect that $\operatorname{dim} \mathbb{C}_{0}>0$.

\section{B. Construction of the contact matrix}

Suppose that particle $i$ and $j$ touch at contact $\alpha$. Then one can define a unit vector $\hat{n}_{\alpha}$ normal to the particles' surfaces at the contact:

$$
\hat{n}_{\alpha}=\frac{\vec{r}_{i}-\vec{r}_{j}}{\left|\vec{r}_{i}-\vec{r}_{j}\right|}
$$

To discuss tangential forces, we must define a unit tangential vector such that $\hat{n}_{\alpha} \cdot \hat{t}_{\alpha}=0$. Given $\hat{n}_{\alpha}$, there are two possibilities for $\hat{t}_{\alpha}$, but $\hat{t}_{\alpha}$ can be uniquely defined by imagining that the two dimensional space is embedded in three dimensions, and writing

$$
\hat{t}_{\alpha}=\hat{n}_{\alpha} \times \hat{z}
$$

where $\hat{z}$ is the unit vector, pointing upwards, perpendicular to the two-dimensional plane.

With these definitions, it is now possible to write the forces $\Delta \vec{f}_{i \alpha}$ and $\Delta \vec{f}_{j \alpha}$ due to contact $\alpha$ on particles $i$ and $j$ :

$$
\Delta \vec{f}_{i \alpha}=R_{\alpha} \hat{n}_{\alpha}+T_{\alpha} \hat{t}_{\alpha}, \quad \Delta \vec{f}_{j \alpha}=-R_{\alpha} \hat{n}_{\alpha}-T_{\alpha} \hat{t}_{\alpha},
$$

But this notation is awkward, because it is necessary to distinguish the two particles of the contact. One of the particles is "first" (particle $i$ ) and the other is "second" (particle $j$ ). The choice of which particle is first is arbitrary, but once the choice is made, it must not be changed. Accordingly, we introduce the symbol $\chi_{i \alpha}$ defined by

$\chi_{i \alpha}=\left\{\begin{array}{cl}1 & \text { if particle } i \text { is first in contact } \alpha, \\ -1 & \text { if particle } i \text { is second in contact } \alpha, \\ 0 & \text { if particle } i \text { does not participate in contact } \alpha .\end{array}\right.$

For each contact between two grains, one element of $\chi$ is 1 , and another is -1 . Contacts between a wall and a grain contribute only one nonzero element to $\chi$. Using the $\chi$ symbol, Eqs. (11) as a single equation:

$$
\Delta \vec{f}_{k \alpha}=\chi_{k \alpha}\left(R_{\alpha} \hat{n}_{\alpha}+T_{\alpha} \hat{t}_{\alpha}\right) .
$$

This equation holds for $1 \leq k \leq M$, so the total force on a particle can be written as a sum over all the contacts:

$$
\overrightarrow{f_{k}}=\sum_{\alpha=1}^{M} \chi_{k \alpha}\left(R_{\alpha} \hat{n}_{\alpha}+T_{\alpha} \hat{t}_{\alpha}\right)
$$

This equation can be cast in the form of a matrix multiplication. From Eq. (14) and an analogous equation for the torques, it is possible to deduce the components of $\mathbf{c}$. c is a $N \times M$ matrix of submatrices $c_{i \alpha}$, where

$$
c_{i \alpha}=\left(\begin{array}{cc}
\chi_{i \alpha} \hat{n}_{\alpha x} & \chi_{i \alpha} \hat{t}_{\alpha x} \\
\chi_{i \alpha} \hat{n}_{\alpha y} & \chi_{i \alpha} \hat{t}_{\alpha y} \\
0 & \left|\chi_{i \alpha}\right| r_{i}
\end{array}\right)
$$

\section{The contact conditions}

Eq. (5) does not give a complete description of motionless granular packings. Granular packings are nonlinear because only certain contact forces are physically possible. For dry granular materials with Coulomb friction, two conditions need to be met:

$$
R_{\alpha} \geq 0, \text { and }\left|T_{\alpha}\right| \leq \mu R_{\alpha},
$$

for $\alpha=1, \ldots M$. The first condition says that there are no attractive forces, only repulsive ones. The second condition states that the tangential force cannot exceed $\mu$ times the normal force, where the constant $\mu$ is the Coulomb friction ratio. Let us define $\mathbb{K}$ to be the set of all contact states satisfying Eq. (16). In Fig. 1] we show the cross section of $\mathbb{K}$, cut by the $(R, T)$ plane of a contact.

Later in this paper, our calculations will be greatly simplified because $\mathbb{K}$ is a convex set. This can be seen from Fig. 1 since $\mathbb{K}$ is a cone with its vertex at the origin. The convexity of $\mathbb{K}$ can also established by a simple proof. Consider two points $\mathbf{F}_{A}, \mathbf{F}_{B} \in \mathbb{K}$. The points

$$
\mathbf{F}_{\lambda}=\lambda \mathbf{F}_{A}+(1-\lambda) \mathbf{F}_{B}, \quad 0 \leq \lambda \leq 1 .
$$




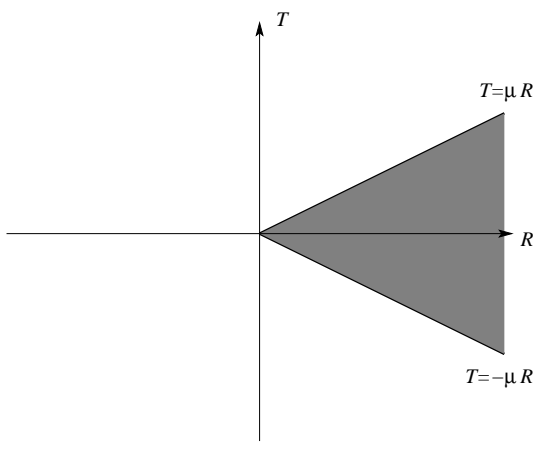

FIG. 1: Cross section of $\mathbb{K}$ cut by the $(R, T)$ of a contact. Contacts that satisfy Eq. (16) must lie in the shaded triangular region.

lie on a straight line between $\mathbf{F}_{A}$ and $\mathbf{F}_{B}$. One can show that $\mathbf{F}_{\lambda} \in \mathbb{K}$ as well, showing that $\mathbb{K}$ is convex.

As we shall see, contacts where an equality holds in Eq. (16), i.e., where $R_{\alpha}=0$ or $\left|T_{\alpha}\right|=\mu R_{\alpha}$, play a special role in limiting the indeterminacy. Contacts with $R_{\alpha}=0$ are called "non-transmitting" contacts because the particles touch, but exert no force on each other. Contacts where $\left|T_{\alpha}\right|=\mu R_{\alpha}$ holds will be called "sliding contacts" even if there is no relative motion. This term is used because this equality holds when two particles slide relative to one another.

\section{The set of possible contact states}

We are now in a position to find the contact forces in a static granular packing and understand how indeterminacy arises. Given an arrangement of particles and external forces acting on each particle (e.g. gravity), one can calculate the contact matrix $\mathbf{c}$ and the vector $\mathbf{M g}$. Then one first searches for a "particular solution" $\mathbf{F}_{1}$ such that $\mathbf{c F}_{1}=-\mathbf{M g}$. These forces are necessary to cancel the external forces. The particular solution $\mathbf{F}_{1}$ can be made unique by requiring that it be orthogonal to every vector in $\mathbb{C}_{0}$.

In general, $\mathbf{F}_{1}$ will not obey the contact conditions Eq. (16), so one must find some $\mathbf{F}_{0} \in \mathbb{C}_{0}$ such that

$$
\mathbf{F}_{0}+\mathbf{F}_{1} \in \mathbb{K}
$$

The sum $\mathbf{F}_{0}+\mathbf{F}_{1}$ is a possible contact state. There could be many vectors $\mathbf{F}_{0} \in \mathbb{C}_{0}$ that satisfy Eq. (18), so the solution may not be unique. On the other hand, not all vectors from $\mathbb{C}_{0}$ will satisfy Eq. (18).

Let $\mathbb{F}$ be the set of all such contact states $\mathbf{F}=\mathbf{F}_{0}+\mathbf{F}_{1}$ satisfying Eq. (18). Then $\mathbb{F}$ is the set of all contact states that could be observed in a given granular packing. We can summarize the requirements of a contact state by writing

$$
\mathbb{F}=\left\{\mathbf{F}_{0}+\mathbf{F}_{1} \mid \mathbf{F}_{0}+\mathbf{F}_{1} \in \mathbb{K}, \mathbf{F}_{0} \in \mathbb{C}_{0}, \mathbf{c} \mathbf{F}_{1}=\mathbf{M g}\right.
$$

$$
\left.\mathbf{F}_{1} \cdot \mathbf{F}_{0}^{(i)}=0 \text { for } i=1 \ldots \operatorname{dim} \mathbb{C}_{0}\right\}
$$

Here $\left\{\mathbf{F}_{0}^{(i)}, i=1 \ldots \operatorname{dim} \mathbb{C}_{0}\right\}$ denotes a basis of $\mathbb{C}_{0}$. In Ref. [12], $\mathbb{F}$ is called the "force network ensemble".

It is clear that the set $\mathbb{F}$ is also convex. Let $\mathbf{F}_{a}$ and $\mathbf{F}_{b}$ be members of $\mathbb{F}$. Then the intermediate points $\mathbf{F}_{\lambda}$ have the form

$$
\begin{aligned}
\mathbf{F}_{\lambda} & =\lambda \mathbf{F}_{a}+(1-\lambda) \mathbf{F}_{b}=\mathbf{F}_{1}+\lambda \mathbf{F}_{0, a}+(1-\lambda) \mathbf{F}_{0, b} \\
& =\mathbf{F}_{1}+\mathbf{F}_{0, \lambda},
\end{aligned}
$$

where we have used the decompositions $\mathbf{F}_{a}=\mathbf{F}_{1}+\mathbf{F}_{0, a}$ and $\mathbf{F}_{b}=\mathbf{F}_{1}+\mathbf{F}_{0, b}$. It can be shown that $\mathbf{F}_{\lambda}$ satisfies all the conditions in Eq. (19): $\mathbf{F}_{\lambda} \in \mathbb{K}$ because $\mathbb{K}$ is convex and $\mathbf{F}_{0, \lambda} \in \mathbb{C}_{0}$ because $\mathbb{C}_{0}$ is a linear subspace. The convexity of $\mathbb{F}$ was first noted in Ref. [15], and shown in Ref. 14].

If $\mathbb{F}$ is empty, the granular packing is unstable, and the particles will move. If $\mathbb{F}$ contains only one point, there is a unique contact state, and there is no indeterminacy. Finally, $\mathbb{F}$ can contain many points. In this case, there is no unique solution.

\section{E. The Boundary of $\mathbb{F}$}

The boundary of $\mathbb{F}$ is analogous to the "yield surface" in the elastoplasticity theory of soils [17]. If a system crosses the boundary, and leaves $\mathbb{F}$, the packing is no longer stable, and the particles will move. When this happens, a new contact matrix must be constructed, including perhaps new contacts, and $\mathbb{F}$ and $\mathbb{C}_{0}$ will change. For our purposes, we are interested in the boundary of $\mathbb{F}$ because it contains the extremal points, where the contact forces are maximized or minimized.

To investigate the structure of $\mathbb{F}$, we will use the concept of an $n$ dimensional neighborhood of point. We say a point $\mathbf{F} \in \mathbb{F}$ has an $n$ dimensional neighborhood if there exist at most $n$ linearly independent vectors $\mathbf{V}$ such that one can find $a_{\min }<0$ and $a_{\max }>0$ satisfying

$$
\mathbf{F}+a \mathbf{V} \in \mathbb{F}, \text { for all } a_{\min }<a<a_{\max } .
$$

As an example, consider a line segment embedded in two dimensional space. Each point on the line segment has a 1 dimensional neighborhood, and the end points have 0 dimensional neighborhoods.

Now let us apply this concept to our set $\mathbb{F}$. Let us suppose that that $\mathbb{F}$ contains a contact state $\mathbf{P}_{0}$ with no sliding or non-transmitting contacts. A multiple of any vector in $\mathbb{C}_{0}$ can be added to $\mathbf{P}_{0}$, as long as it is small enough. Therefore, there are $n=\operatorname{dim} \mathbb{C}_{0}$ linearly independent vectors $\mathbf{V}$ satisfying Eq. (21), meaning that $\mathbf{P}_{0}$ has an $n$ dimensional neighborhood.

Next suppose that we have a point $\mathbf{P}_{1} \in \mathbb{F}$ with exactly one sliding contact. Let us label that sliding contact $\beta$ and assume that we have $\mu R_{\beta}^{(1)}=T_{\beta}^{(1)}$. Points $\mathbf{P}_{a}=$ $\mathbf{P}_{1}+a \mathbf{V}$ obey

$$
\mu R_{\beta}^{(a)}-T_{\beta}^{(a)}=\mu R_{\beta}^{(1)}-T_{\beta}^{(1)}+a\left(\mu R_{\beta}^{(V)}-T_{\beta}^{(V)}\right),
$$


where $R_{\beta}^{(a)}, R_{\beta}^{(1)}$, and $R_{\beta}^{(V)}$ are the appropriate components of $\mathbf{P}_{a}, \mathbf{P}_{1}$, and $\mathbf{V}$ respectively. Since contact $\beta$ is sliding in the state $\mathbf{P}_{1}$, we have $\mu R_{\beta}^{(1)}-T_{\beta}^{(1)}=0$. Now, note that $a$ takes on both positive and negative values. If $\mathbf{P}_{a}$ is to satisfy the contact conditions, we also need $\mu R_{\beta}^{(V)}-T_{\beta}^{(V)}=0$, i.e., contact $\beta$ must be sliding in $\mathbf{V}$ also. Thus the sliding contact puts a constraint on the vectors $\mathbf{V}$ that can be used. However, given any two vectors from $\mathbb{C}_{0}$, one can construct a linear combination satisfying this constraint. In this way, $n-1$ linearly independent vectors can be constructed, so $\mathbf{P}_{1}$ has an $n-1$ dimensional neighborhood. Similar reasoning can be extended to show that a contact state with two sliding contacts has an $n-2$ dimensional neighborhood, and finally, a contact state with $n$ sliding contacts is a 0 dimensional neighborhood, that is, it is an extremal point.

So far, non-transmitting contacts have not been considered. But it is easy to incorporate them, because they can be considered as two sliding contacts superimposed on each other, i.e., the contact obeys $T=\mu R$ and $T=-\mu R$ at the same time.

\section{F. Quantifying indeterminacy}

The indeterminacy of the granular packing is determined by the size and shape of the set $\mathbb{F}$. Since $\mathbb{F}$ is a convex set with a finite number of extremal points, one possible approach would be to locate all its extremal points, but the large number of such points makes this unfeasible. Therefore, we adopt an alternative approach. We locate the subset $\mathbb{F}_{\text {ext }}$ of extremal points where a component of some contact force attains its maximum or minimum possible value. Specifically, for each contact $\alpha$, four different extremal points are found: the state where $R_{\alpha}$ is maximum, then where $R_{\alpha}$ is minimized, and then the states where $T_{\alpha}$ is maximized and then minimized. (Note that minimizing a normal force means making it approach 0 as closely as possible, but minimizing a tangential force means making it approach $-\infty$.)

Once $\mathbb{F}_{\text {ext }}$ has obtained, different measures of indeterminacy can be extracted. One possibility is to calculated the range of possible forces that a given contact $\alpha$ can take on:

$$
\delta_{R, \alpha}=\frac{R_{\max , \alpha}-R_{\min , \alpha}}{\bar{m} g}, \quad \delta_{T, \alpha}=\frac{T_{\max , \alpha}-T_{\min , \alpha}}{\bar{m} g},
$$

where $R_{\max , \alpha}$ and $R_{\min , \alpha}$ are the maximum and minimum possible values of the normal contact force at contact $\alpha$. To obtain a dimensionless number, we divide by $\bar{m} g$, the average weight of a particle. We call $\delta_{R}$ and $\delta_{T}$ the "local indeterminacies" because they quantify the ambiguity of the force at one contact.

One contact force cannot be maximized independently of the others. One could therefore ask how much the entire contact force state must change when we bring one contact force from its minimum value to its maximum.
As an alternative to $\delta_{R}$ and $\delta_{T}$, one could calculate

$$
\begin{aligned}
& d_{R, \alpha}=\frac{\left\|\mathbf{F}_{R, \max , \alpha}-\mathbf{F}_{R, \min , \alpha}\right\|}{\bar{m} g}, \\
& d_{T, \alpha}=\frac{\left\|\mathbf{F}_{T, \text { max }, \alpha}-\mathbf{F}_{T, \min , \alpha}\right\|}{\bar{m} g} .
\end{aligned}
$$

Here, $\mathbf{F}_{R, \max , \alpha}$ is the contact state where the normal force is maximized at contact $\alpha$, and the other contact states in Eq. (24) are defined analogously. These quantities estimate the "diameter" of $\mathbb{F}$. Note that $\mathbb{F}$ is embedded in a $2 M$ dimensional space, so that $2 \mathrm{M}$ different "diameters" can be calculated. We call $d_{R}$ and $d_{T}$ the "global indeterminacies".

\section{G. Algorithm}

We now give the algorithm used to find the maximum and minimum possible forces at a given contact. The first step is to locate an extremal point. Let us suppose that we begin with a point with an $n$-dimensional neighborhood in $\mathbb{F}$. Given this point, an extremal point can be found in the following way: Pick one of the $n$ vectors from the basis of $\mathbb{C}_{0}$. Then, starting from the interior point, move in that direction until a sliding contact is detected. Then $n-1$ linearly independent vectors can be constructed out of the basis of $\mathbb{C}_{0}$, all of which preserve the status of the sliding contact. Pick one of these vectors, and proceed in its direction until a second sliding contact is detected (or the first sliding contact becomes non-transmitting). Then $n-2$ linearly independent vectors can be built which obey these two constraints. Continuing in this way, we will eventually reach a point where there are $n$ constraints, and no vectors can be constructed that respect all of them. This is an extremal point.

Once the extremal point has been reached, its neighboring extremal points can each be identified. Recall that an extremal point is characterized by $n$ constraints arising from $n$ sliding contacts. If we relax one of these constraints, there is one direction in $\mathbb{C}_{0}$ that respects all the other $n-1$ constraints. If we move away from our extremal point in this direction, we will eventually encounter a new extremal point. This is a neighboring extremal point, connected to the current point by an edge. Since there are $n$ possible constraints to relax, each extremal point will have $n$ neighbors. Each of these neighbors can be checked. If none of them are "better" than the current point (in the sense that the relevant contact force is greater or less), then the current point is the best point. If any one of the neighboring points is better, we move there and repeat the process. The convex structure of $\mathbb{F}$ guarantees that there are no local minima or maxima that would trap the algorithm.

The algorithm must deal with a number of practical difficulties. For example, it can happen that a contact must always be sliding or non-transmitting. It is necessary to detect this, because such a situation reduces the dimension of $\mathbb{F}$. Therefore before beginning to search for 


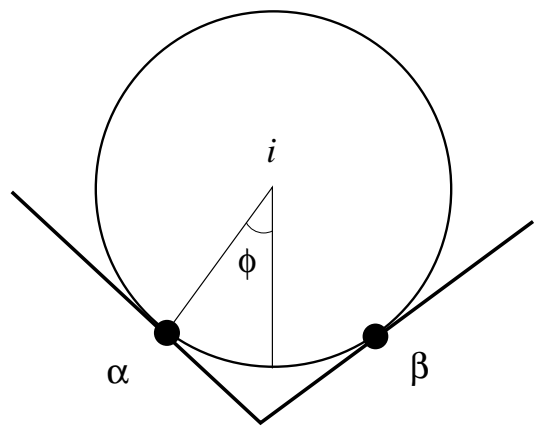

FIG. 2: The particle $i$ is supported by two walls through contacts $\alpha$ and $\beta$. Gravity pulls the particle downwards. The angle $\phi$ suffices to characterize the geometry of this simple granular packing.

extremal points, we first try to locate a point without sliding contacts. This can be difficult, because the simulations often yield points with many sliding contacts. But given such a point, one can construct a vector belonging to $\mathbb{C}_{0}$ that preserves all the sliding contacts except one, and then moving along this vector so that the number of sliding contacts is reduced by one. This process can be repeated. Sometimes this does not work, because the simulation yields a point in a tight, multi-dimensional corner. In this case, the contact dynamics iterative solver can be used to generate an alternative starting point.

In about $1 \%$ of the cases analyzed in Sec III extremal states are found with a different number of sliding contacts than expected. This may happen when one constructs linear combinations that satisfy a given constraint, and by chance, satisfy several other constraints at the same time. Another possibility is that it is difficult to maintain sufficient numerical accuracy during the construction of the linear combinations. Recall that one cannot test strict equalities with floating-point numbers; one should always check that equality conditions such as $T= \pm \mu R$ are satisfied to a certain tolerance. This may cause an occasional overestimate of the number of sliding contacts. However, since this situation occurs only rarely, it does not affect the conclusions of this paper.

\section{H. Two Contacts}

As a simple application of these ideas, consider the granular packing in Fig 2 where $M=2$ and $N=1$. The equations of static equilibrium are

$$
\begin{aligned}
R_{\alpha} \sin \phi+T_{\alpha} \cos \phi-R_{\beta} \sin \phi+T_{\beta} \cos \phi & =0, \\
R_{\alpha} \cos \phi-T_{\alpha} \sin \phi+R_{\beta} \cos \phi+T_{\beta} \sin \phi & =m g, \\
r T_{\alpha}+r T_{\beta} & =0 .
\end{aligned}
$$

Comparing this to Eq. (5), we have

$$
\mathbf{c}=\left(\begin{array}{cccc}
\sin \phi & \cos \phi & -\sin \phi & \cos \phi \\
\cos \phi & -\sin \phi & \cos \phi & \sin \phi \\
0 & r & 0 & r
\end{array}\right) .
$$

This matrix has a null space that has at least one dimension. Let us find $\mathbf{F}_{0}$ by solving the system of equations $\mathbf{c F}_{0}=0$. The result is

$$
\mathbf{F}_{0}=\left(\begin{array}{c}
\sin \phi \\
\cos \phi \\
\sin \phi \\
-\cos \phi
\end{array}\right)
$$

Note that $\mathbf{F}_{0}$ corresponds to the horizontal components of the contact forces canceling each other.

The particular solution can be found by solving $\mathbf{c F}_{1}=$ $\mathbf{M g}$, and then requiring that $\mathbf{F}_{0} \cdot \mathbf{F}_{1}=0$. The result is

$$
\mathbf{F}_{1}=\frac{m g}{2}\left(\begin{array}{c}
\cos \phi \\
-\sin \phi \\
\cos \phi \\
\sin \phi
\end{array}\right)
$$

which simply expresses the requirement that the vertical component of the contact forces cancel gravity. The contact states in $\mathbb{F}$ all have the form

$$
\mathbf{F}_{1}+a \mathbf{F}_{0} .
$$

Applying the contact conditions puts restrictions on the values of $a$ which are allowed. For example, requiring $R_{\alpha} \geq 0$ and $R_{\beta} \geq 0$ means that $a$ must satisfy the inequality

$$
a \geq-\frac{m g}{2} \cot \phi
$$

And the Coulomb condition becomes

$$
\mu \frac{m g}{2} \cos \phi+\mu a \sin \phi \leq\left|-\frac{m g}{2} \sin \phi+a \cos \phi\right| .
$$

Working out the various cases connected with the absolute values, one obtains a lower bound for $a$.

$$
a \geq a_{\min }=-\frac{m g}{2}\left(\frac{\mu-\tan \phi}{1+\mu \tan \phi}\right) .
$$

If this condition is fulfilled, than Eq. (30) is always satisfied as well. When $\tan \phi<1 / \mu$, there is an upper bound for $a$ :

$$
a \leq a_{\max }=\frac{m g}{2}\left(\frac{\mu+\tan \phi}{1-\mu \tan \phi}\right) .
$$

If this condition is fulfilled, then Eq. (30) is satisfied as well. When $\tan \phi>1 / \mu$, there is no upper bound on $a$; $a$ can be arbitrarily large.

Now the indeterminacy of this packing can be calculated. Selecting the appropriate components from Eq. (29), we have

$$
R_{\alpha}=R_{\beta}=\frac{m g}{2}(\cos \phi+a \sin \phi) .
$$

The maximum and minimum possible forces can be obtained by setting $a$ equal to $a_{\max }$ or $a_{\min }$ respectively. When this is done, one obtains

$$
\delta_{R}=\frac{R_{\max }-R_{\min }}{m g}=\frac{a_{\max }-a_{\min }}{m g} \sin \phi
$$




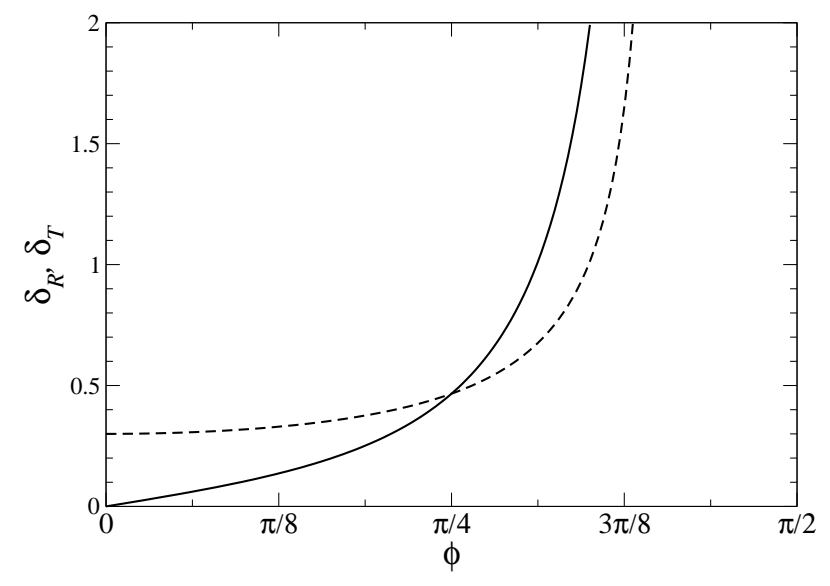

FIG. 3: The measures of indeterminacy $\delta_{R}$ (solid line) and $\delta_{T}$ (dotted line). $\phi$ is the angle shown in Fig. 2 and $\mu$ was taken to be 0.3. At $\phi=0, \delta_{1}=\mu$ and $\delta_{2}=2 \mu$. Both measures diverge at $\tan \phi=1 / \mu$ or $\phi \approx 0.41 \pi$.

$$
=\frac{\mu \sin \phi}{\cos ^{2} \phi-\mu^{2} \sin ^{2} \phi} .
$$

In the same way, one has

$$
-T_{\alpha}=T_{\beta}=\frac{m g}{2}(\sin \phi-a \cos \phi) .
$$

Inserting $a=a_{\max }$ and $a=a_{\min }$, we obtain

$$
\delta_{T}=\frac{\mu \cos \phi}{\cos ^{2} \phi-\mu^{2} \sin ^{2} \phi} .
$$

The behavior of Eqs. (35) and (37) are shown in Fig. 3

\section{NUMERICAL APPLICATION}

\section{A. Overview}

We have investigated numerically the indeterminacy of granular packings with $N=95$ particles. Fig. 4 shows the procedure used to generate the various configurations considered in this section. First, $N=95$ grains were placed on a grid inside a rectangular box of size $L_{x} \times L_{y}$. To prevent the formation of regular arrays of particles, the radii of the particles are uniformly distributed in the interval $\left[0.7 r_{\max }, r_{\max }\right]$. Then, the grains were allowed to fall, under the influence of gravity, and form a packing at the bottom of the box. This process was simulated by both $\mathrm{CD}$ and MD, yielding the CD-1 and MD-1 configurations. Both simulations continue until the kinetic energy decreases to a negligible value, or the elapsed time reaches $6 \sqrt{L_{y} / g}$, i.e. about twice the time a particle needs to fall a distance $L_{y}$. (This second condition is needed because occasionally a particle falls to the bottom and starts to roll with a small amount of kinetic energy that is enough to violate the first condition, but

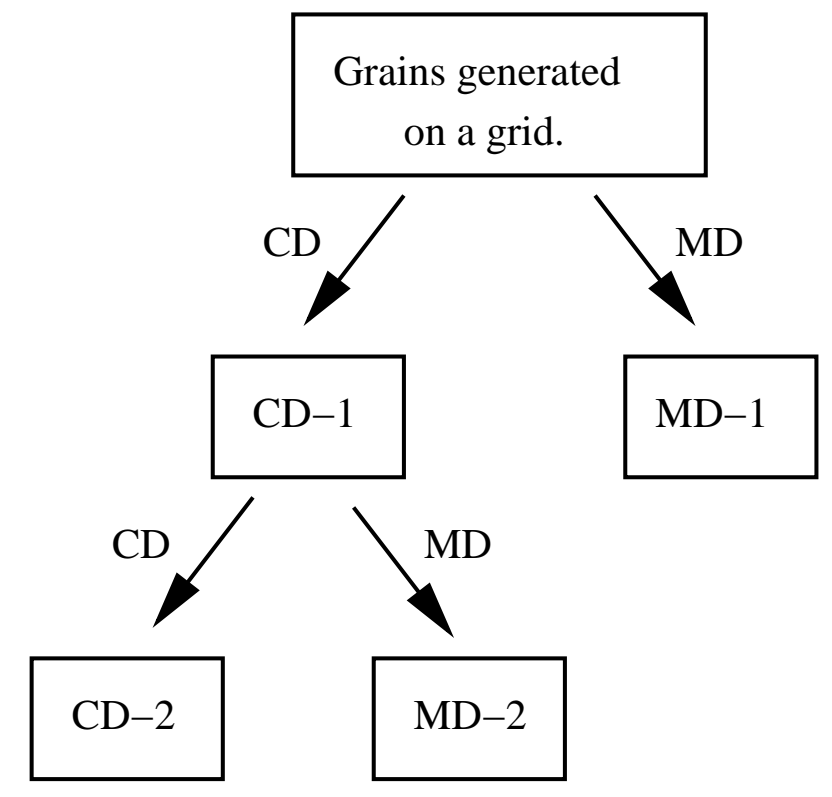

FIG. 4: A diagram showing the relationship between the various configurations analyzed in this section. First, 95 grains were placed on a rectangular grid. Then, the grains were allowed to fall, under gravity, and settle at the bottom of the container. This process was simulated by both CD and MD, yielding the CD-1 and MD-1 configurations. Then the CD-1 configuration was taken as the initial condition for two more simulations, yielding CD-2 and MD-2.

not large enough so that it reaches another particle or a wall in a reasonable amount of time.) Then the CD-1 configuration is used as the starting point for two more simulations, yielding the CD-2 and MD-2 configuration, according to the simulation method used. In this second CD simulation, the initial guess for the contact forces is $\mathbf{F}=0$. This procedure was repeated sixty times, yielding a set of 240 configurations. The 60 initial conditions are distinguished by choosing different particle radii each time.

In the MD simulations, particles interact via linear, damped springs in both the normal and tangential directions. When a contact becomes sliding, it is assumed that the tangential spring remains stretched at its maximum length. The springs have stiffness $1.2 \times 10^{5} \bar{m} g / r$ and damping constant $7.7 \times 10^{4}(\bar{m} g / r) \sqrt{r / g}$. This choice of parameters leads to an average particle overlap of $7 \times 10^{-6}$ of a particle radius. Usually particles are much softer in MD simulations, but extremely hard particle were used here to approach as closely as possible the CD simulations (average overlap: $6 \times 10^{-8}$ of a particle radius). Due to the hardness of the particles, the MD simulations were very slow, taking roughly 100 times as long as the $\mathrm{CD}$ ones. In the CD simulations, the normal and tangential resitution coefficients were set to 0 . In all cases, the Coulomb friction ratio was $\mu=0.3$.

Then the resulting configurations are analyzed. The 

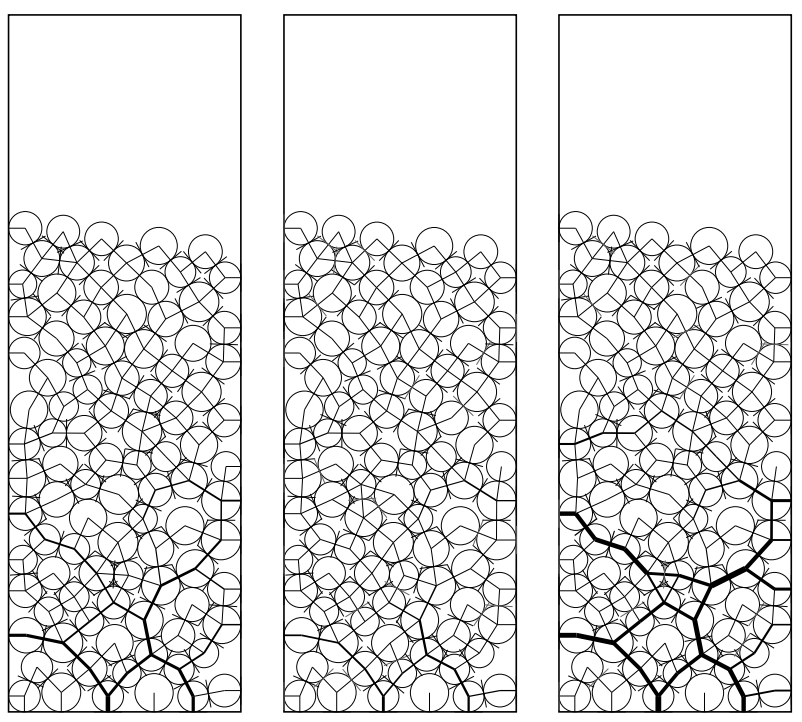

FIG. 5: Three different contact states for a configuration with $N=95$ particles. The CD-1 state obtained from contact dynamics is on the left. There are $M=160$ contacts. Then this configuration was allowed to relax in a molecular dynamics simulation, resulting in the MD-2 configuration shown in the middle. During the relaxation, the number of contacts increases to $M=166$. The state on the right is the extremal state of the CD-1 configuration with the maximum norm. The thickness of the lines connecting the centers is proportional to the normal force, and tangential forces are shown by lines tangent to the particle surfaces.

matrix c was constructed, and a singular value decomposition was used to extract its null space. Then the set $\mathbb{F}_{\text {ext }}$ defined in Sec. IIF is found using the algorithm presented in Sec. IIG Some examples of the contact states found are shown in Fig. [5] The left hand panel is the CD1 state, the middle panel is the MD-2 state, right hand panel shows one of the elements of $\mathbb{F}_{\text {ext }}$. One can see already that all three states are different, but the CD-1 and MD-2 states are closer together than either one with the extremal state.

Fig. 67 shows the dimension of $\mathbb{F}$ as a function of the number of contacts. All points fall onto or just above the line $\operatorname{dim} \mathbb{F}=2 M-3 N$, confirming the prediction that $\operatorname{dim} \mathbb{F} \geq 2 M-3 N$. Fig. 6] also shows that $\operatorname{dim} \mathbb{F}$ never exceeds $2 M-3 N$ by more than 3 . This means that it is reasonable to use $\operatorname{dim} \mathbb{F} \approx 2 M-3 N$ to estimate $\operatorname{dim} \mathbb{F}$. There is also no difference between the classes of configurations, except that MD simulations tend to have more contacts than the CD ones.

Fig. 6] shows the number of sliding contacts observed in the different configurations. A contact is considered sliding if $\mu R-|T|<\epsilon \bar{m} g$. For Fig. 6b,$\epsilon=10^{-9}$. Nontransmitting contacts $(R,|T|<\epsilon \bar{m} g)$ are counted as two sliding contacts. The number of sliding contacts is always less than or equal to $2 M-3 N$, consistent with our analysis of the boundary of $\mathbb{F}$ in Sec.IIE The difference
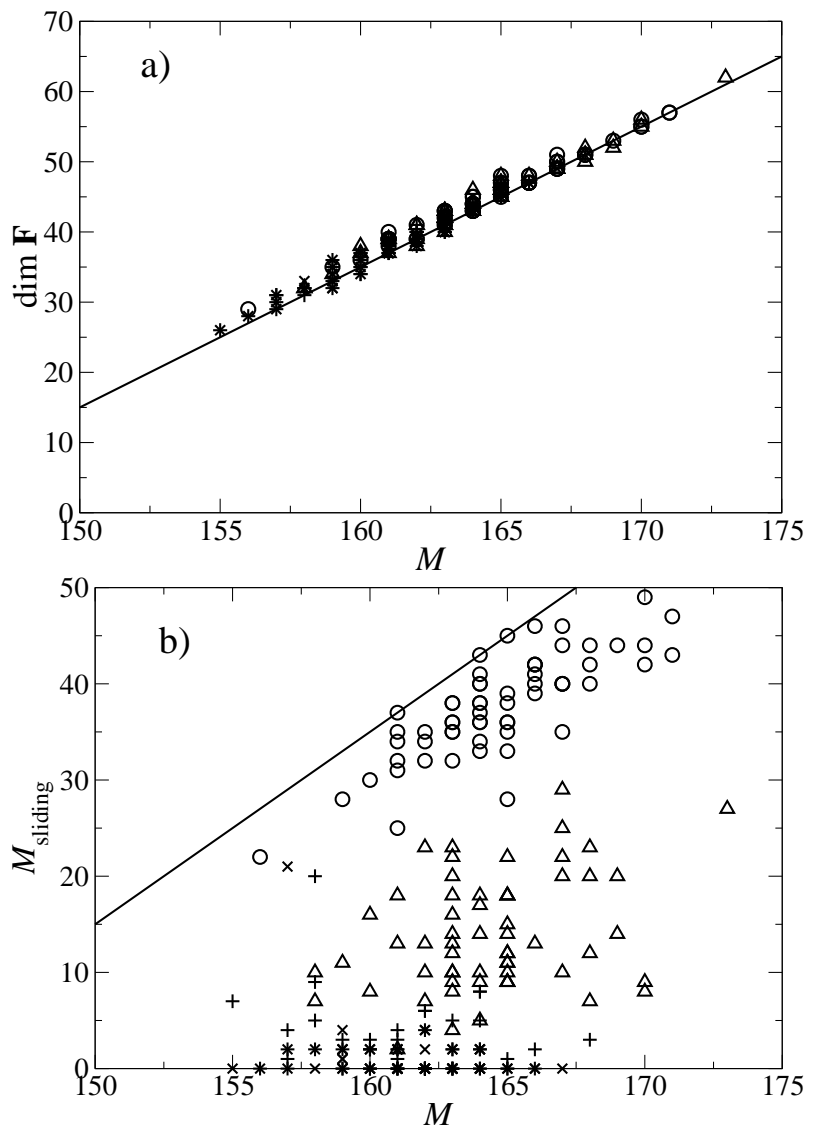

FIG. 6: (a) the dimension of the set of possible contact states $\mathbb{F}$ as a function of the number of contacts $M$. The solid line shows the lower $\operatorname{limit} \operatorname{dim} \mathbb{F}=2 M-3 N$. (b) the number $M_{s}$ of sliding contacts as a function of $\operatorname{dim} \mathbb{F}$. The straight line shows $M_{s}=2 M-3 N$. A contact is considered sliding if $\mu R-|T|<$ $\epsilon \bar{m} g$, with $\epsilon=10^{-9}$. Non-transmitting contacts $(R, T<\epsilon \bar{m} g)$ count as two sliding contacts, consistent with our discussion in Sec. IIE Four different families of simulations are shown: triangles - MD-1, circles - MD-2, crosses - CD-1, x's - CD-2.

between the different classes of configurations becomes clear. The CD simulations have very few sliding contacts. The two classes of MD simulations are also well separated from each other, with the MD-2 configurations having the most sliding contacts. This difference between the two configurations shows that the MD simulation is able to retain a memory of how it was formed. The MD1 simulation was generated by letting the particles fall from a given height, whereas the MD-2 simulation was started with the particles almost in their final position. Therefore, much more energy was dissipated during the MD-1 simulation than the MD-2 simulations. The difference in the number of sliding contacts is a sign of their different histories.

Note that the CD simulations lack this kind of memory. A closer examination of the CD-2 simulations reveals that there are initially many sliding contacts, but this number decreases rapidly to values nearly zero, as 


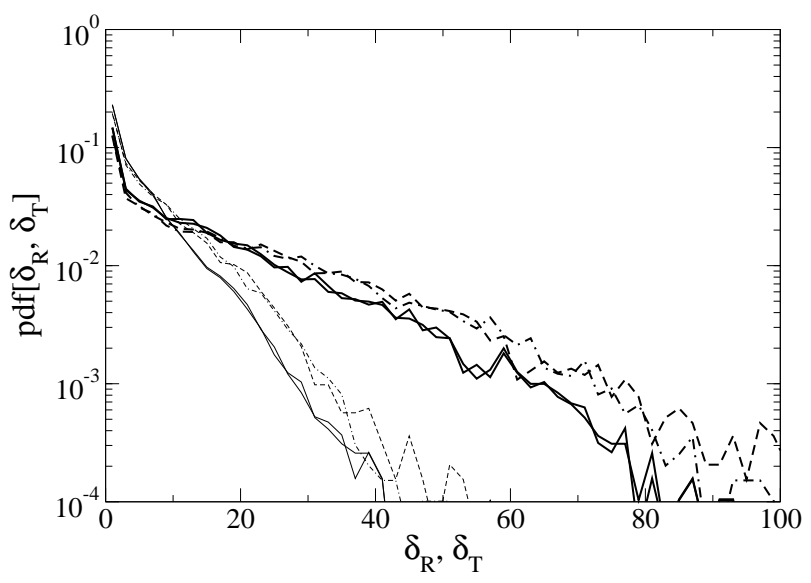

FIG. 7: Histograms of local indeterminacy $\delta_{R}$ (thick lines) and $\delta_{T}$ (thin lines), defined in Eq. (23), for the CD configurations (solid lines), MD configurations (dashed lines), plotted semilogarithmically. The thick lines show $\delta_{R}$ and the thin lines $\delta_{T}$. The distributions are normalized so that their integral is unity, hence we call them probability density functions.

shown in Fig. 6b. There is one exceptional simulation, indicated by the cross near $M_{s} \approx 20, \operatorname{dim} \mathbb{F} \approx 30$, where the system seems to be trapped in some corner of $\mathbb{F}$ and unable to escape. (The nearby $\mathrm{CD}-1$ simulation probably represents a similar situation, but the two points were generated by different random number seeds, so it is probably a coincidence that they are so close together.)

\section{B. Measurement of Indeterminacy}

\section{Local indeterminacy}

The local indeterminacy presented in Sec. IIF can be calculated. In Fig. 7 we show the distribution of $\delta_{R}$ and $\delta_{T}$ for the four different families of configurations. In all cases, the distributions are exponential, except where a sharp peak appears near $\delta_{R}, \delta_{T}=0$. Note that similar exponential tails are observed in contact force distributions.

The MD distributions show slightly larger indeterminacies than the $\mathrm{CD}$ ones, independent of the method of generating the configuration. No contacts were observed with infinite indeterminacies, although it is theoretically possible, as was shown in Sec. IIH] Infinite indeterminacy may exist in only special packings with very small number of particles.

\section{Global indeterminacy}

Let us now consider global indeterminacy. Histograms of the $d_{R}$ and $d_{T}$, defined in Eq. (24), are shown in Fig. 8 for the three different families of configurations. This

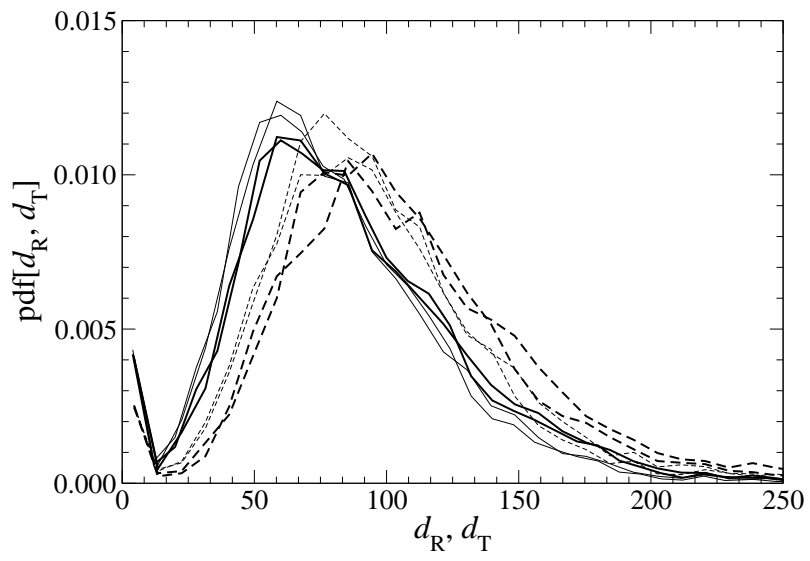

FIG. 8: Histograms of global indeterminacy $d_{R}$ (thick lines) and $d_{T}$ (thin lines), defined in Eq. (24), for the CD simulations (solid lines), and the MD simulations (dashed lines).

measure of indeterminacy has very different properties from the previous one. In Fig. 7 the most probable values of the indeterminacy were small, but in Fig. 8 the probability density function presents two maxima, one close to $d_{R}, d_{T}=0$, and the another well separated from the smallest values. The second maximum is by far the largest, so maximizing or minimizing a contact force usually involves changing many forces throughout the packing, even when the change at the contact in question is small. The maxima in Fig. 8 can be taken as crude estimates of the diameter of $\mathbb{F}$, indicating that $\mathbb{F}$ has a diameter of approximately $60 \bar{m} g$ in the CD simulations and $75 \bar{m} g$ in the MD simulations. Consistent with Fig. 7 the MD simulations show slightly higher indeterminacy than the CD ones. Finally, note that the curves for the normal and tangential components are nearly the same in Fig. 8 but clearly different in Fig. 7

\section{Force Chains}

One of the most remarkable characteristics of granular packings is that most of the force is carried by a small fraction of the contacts, which are organized in linear structures called force chains. Examples of force chains are clearly visible in Fig. 5. This figure also suggests that indeterminacy is also concentrated along force chains it is the force bearing contacts which differ the most between the three panels. We can confirm this impression by dividing the contacts into two different classes, those with above average forces and those with below average forces. In Ref. [16], it was shown that these two populations of contacts have different properties, the former being associated with force chains. (In our case, these two classes do not have exactly the same meaning as in Ref. [16], as the stress is not uniform throughout the packing. Nevertheless, it is still possible for us to isolate the contacts in the force chains, at least in the lower 


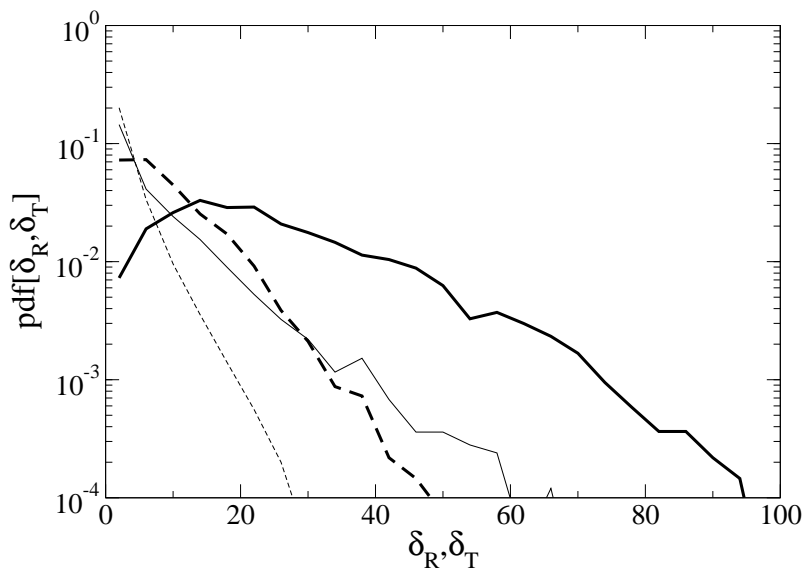

FIG. 9: Probability distributions of the local indeterminacies $\delta_{R}$ (solid lines) and $\delta_{T}$ (dashed lines) for contacts with $R<\bar{R}$ (thin lines), and for $R>\bar{R}$ (thick lines), where $\bar{R}=9.3 \bar{m} g$ is the average normal force. Only data from the CD-1 simulations are shown; the others yield similar curves.

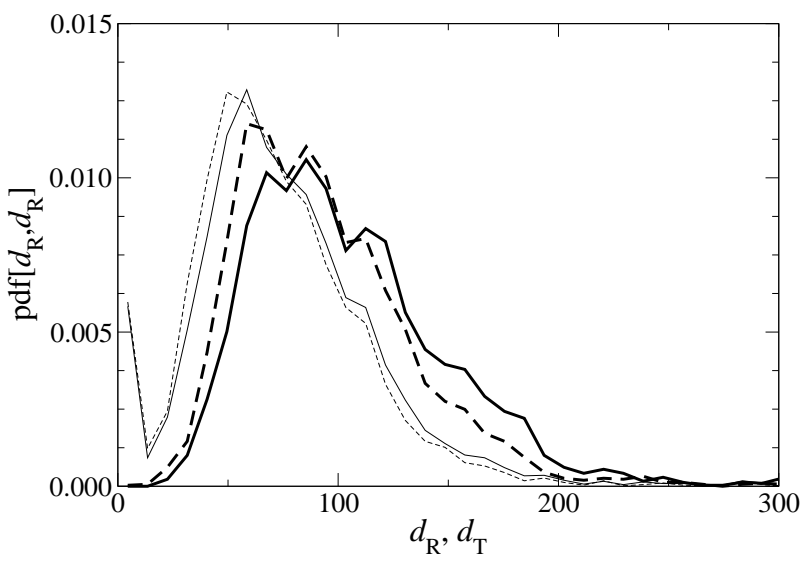

FIG. 10: Probability distributions of the global indeterminacies $d_{R}$ (solid lines) and $d_{T}$ (dashed lines) for contacts with $R<\bar{R}$ (thin line), and for $R>\bar{R}$ (thick line), where $\bar{R}$ is the average normal force. Only data from the CD-1 simulations are shown; the others yield similar curves.

part of the packing.) In Fig. 9] we show the distributions of local indeterminacy in the CD configurations for each class of contact. The two classes yield quite different distributions. The peak at $\delta_{R}, \delta_{T}=0$ is due entirely to the contacts with below average force, while the contacts with large indeterminacy have above average forces. Thus, force chains are also "indeterminacy chains".

We can examine the distribution of global indeterminacy as well. This is done in Fig.10. The small maximum near $d_{R}, d_{T}=0$ is due entirely to contacts with $R<\bar{R}$. The main maximum has contributions from both classes of contacts, but removing the weak contacts causes the maximum to shift towards larger values.

\section{Alternative measurements of indeterminacy}

In Ref. 14], indeterminacy is measured in a different way. A configuration is given to the $\mathrm{CD}$ simulation program, and iterative solver of the CD simulation is asked for a possible solution to the forces. This solution is then perturbed slightly, and given to the iterative solver as an initial guess, and a new solution is obtained. This is repeated many times, and the force network ensemble is sampled in the same way as different statistical mechanical ensembles are sampled in Monte Carlo simulations. Of course, there is no guarantee that that this method will weight appropriately the different regions of $\mathbb{F}$ or even that it will explore all parts of $\mathbb{F}$.

We have carried out this procedure on our packings. For each configuration, we have obtained 500 different solutions. The solutions are perturbed by multiplying all the contact forces by a random number uniformly distributed between 0.5 and 1.5. Then the center $\mathbf{F}_{\circ}$ of $\mathbb{F}$ can be estimated by averaging over all 500 points, and the radius $r$ of $\mathbb{F}$ can be estimated by the variance:

$$
r=\left(\frac{1}{500} \sum_{i=1}^{500}\left[\mathbf{F}_{i}-\mathbf{F}_{\circ}\right]^{2}\right)^{1 / 2} .
$$

Here, $\mathbf{F}_{i}$ are the solutions obtained from the iterative solver. Averaging over all 60 configurations in each class, we obtain $r=(33 \pm 1.4) \bar{m} g$ for the CD-1 configurations, $r=(34 \pm 1.3) \bar{m} g$ for CD-2, $r=(41 \pm 1.7) \bar{m} g$ for MD-1, and $r=(40 \pm 1.5) \bar{m} g$ for MD-2. These values are quite close to half the diameter of $\mathbb{F}$ estimated from Fig. 8 In all cases, the standard deviation of $r$ is about $12 \bar{m} g$, which is also consistent with Fig. 8 This suggests that one can indeed sample $\mathbb{F}$ in this way.

\section{Comparison between $\mathrm{MD}$ and $\mathrm{CD}$}

\section{Distance between the states}

Next, we would like to examine more closely the difference between the forces calculated by MD and CD. One way to do this is to compare the CD-1, CD-2, and MD-2 configurations, where the particles positions are nearly identical. The distance between two states $\mathbf{F}_{A}$ and $\mathbf{F}_{B}$ is simply $\left\|\mathbf{F}_{A}-\mathbf{F}_{B}\right\|$. If $\mathbf{F}_{A}$ and $\mathbf{F}_{B}$ have different numbers of contacts, one can insert 0's into the appropriate places so that they have the same dimension.

We find that the distance between CD-1 and CD-2 is $(50 \pm 3) \bar{m} g$, while $(75 \pm 4) \bar{m} g$ separates CD-1 and MD-2. Thus, the MD moves the forces farther away from the initial state than CD does. But the MD-2 and CD-2 states are separated by only $(45 \pm 3) \bar{m} g$, indicating that they move in approximately the same direction. Note that all these distances are less than, but of approximately the same magnitude as, the diameter of $\mathbb{F}$. Hence changing from $\mathrm{CD}$ to $\mathrm{MD}$ or erasing the memory of $\mathrm{CD}$ causes 

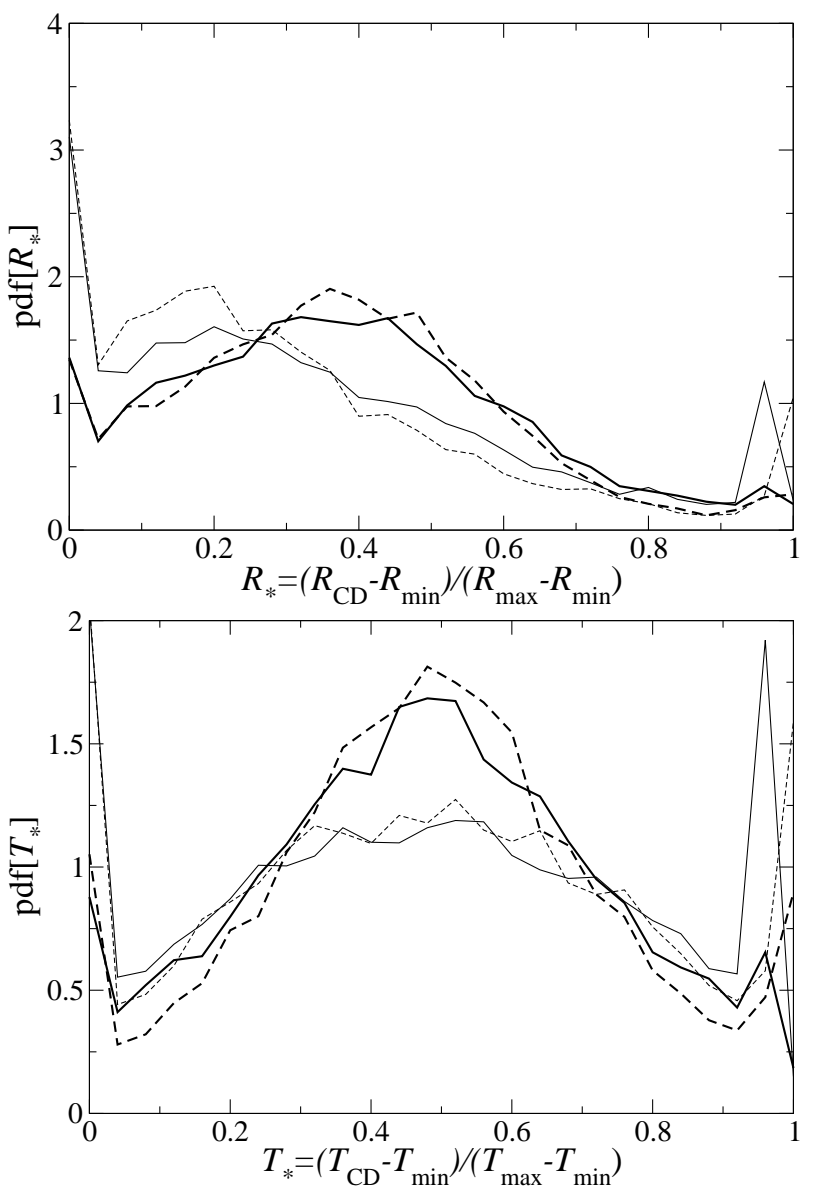

FIG. 11: Histograms of $R_{*}$ and $T_{*}$, defined in Eq. (39), for the four different configurations. The solid lines show CD simulations, and the dotted lines show MD simulations. The thick lines show the results of dropping the particles (CD-1 and MD-1), and the thin lines show the result of obtaining the forces with very little particle movement (CD-2 and MD-2).

a perturbation in the forces of roughly the same size as their indeterminacy.

\section{Relation to extremal states}

To see more precisely where the state found by the simulation stands in relation to the extremal states, let us consider the following quantities:

$$
R_{*}=\frac{R_{\mathrm{sim}}-R_{\min }}{R_{\max }-R_{\min }}, \quad T_{*}=\frac{T_{\mathrm{sim}}-T_{\min }}{T_{\max }-T_{\min }},
$$

where $R_{\text {sim }}$ and $T_{\text {sim }}$ are the contact forces found by the simulation, and $R_{\max }$ and $T_{\max }$ are the maximum values these forces could attain in this configuration, while $R_{\text {min }}$ and $T_{\min }$ are the minimum forces. $R_{*}=0$ means that the simulation chose the minimum possible force while $R_{*}=1$ means that it chose the maximum. In Fig. 11] we show histograms of $R_{*}$ and $T_{*}$ for the four families

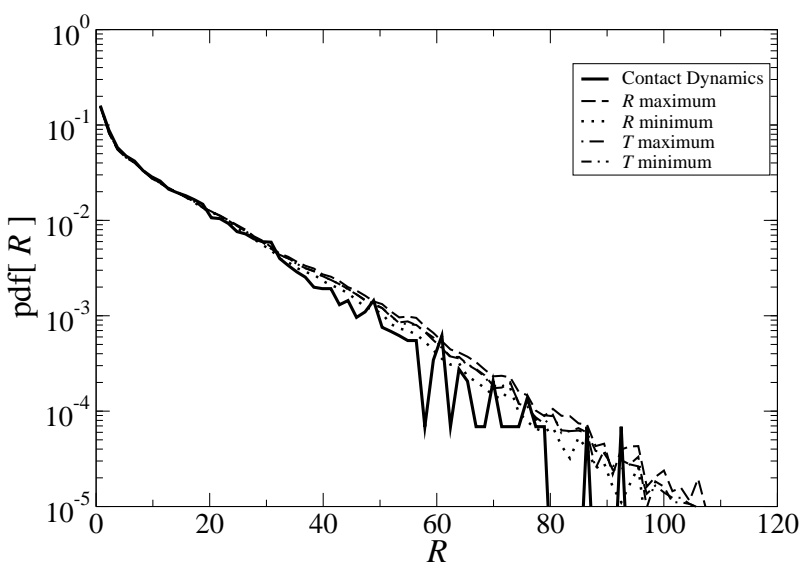

FIG. 12: Histogram of the normal force $R$ in the simulated states and various classes of extremal states. Sixty configurations of $N=95$ particles were considered. The curves for the extremal states have less noise because each configuration contributes $M$ extremal states, but only one contact dynamics state.

of configurations. Surprisingly, these distributions depend more on the history of the configuration than on the simulation method. When the particles are dropped and allowed to settle (CD-1 and MD-1), the normal forces are larger, relative to their minimum and maximum possible values, than when the particles are simply placed into their final positions (CD-2 and MD-2). In the tangential case, the CD-1 and MD-1 states show more values clustered around the middle of the allowed interval than the CD-2 and MD-2 states. On the other hand, the CD-2 and MD-2 states have more contacts at their maximum or minimum values. These results show that the CD method is capable of representing the history of the packing, even though this is not reflected in the number of sliding contacts.

\section{Contact Force distributions}

\section{Extremal vs Simulated states}

The contact force distributions in the various types of contacts states are compared in Fig. 12 The extremal and contact dynamics distributions coincide for $R<30 \bar{m} g$, but then separate, with the extremal states exhibiting more contacts with large forces. This is true, even of extremal states found while minimizing $R$. All the distributions are approximately exponential, similar to those found in other studies 12, 16]. This result suggests that the exponential contact force distributions are a property of all members of $\mathbb{F}$. This means that these exponential tails are probably due to some property of Eq. (5), and can be studied using the force network approach. 


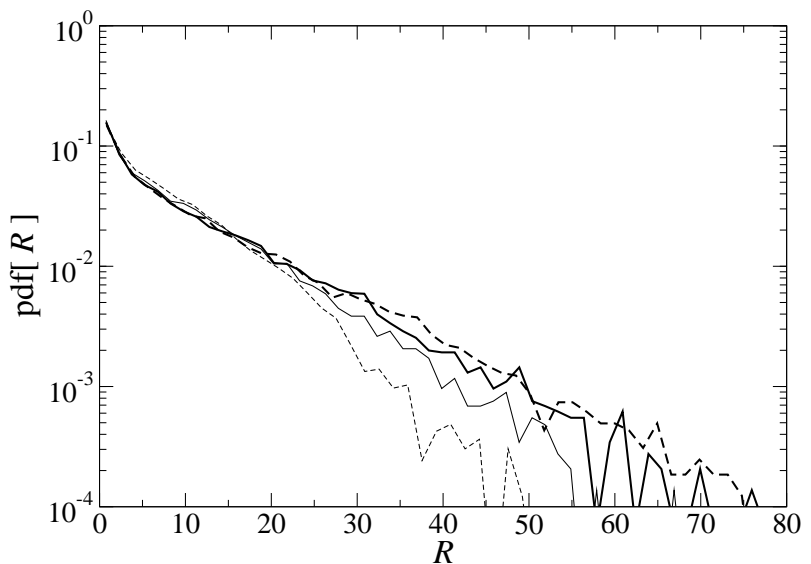

FIG. 13: Histogram of the normal force $R$ for the different families of simulations: CD- 1 - thick solid line, CD- 2 - thin solid line, MD-1 - thick dashed line, MD-2 - thin dashed line.

\section{2. $M D$ vs $C D$}

Finally, we compare in Fig. 13 the normal contact force distributions for the four different families of simulations. Both CD-1 and MD-1 yield similar curves, obeying an exponential fall off out to the largest observed values of $R$. On the other hand, CD-2 and MD-2 have fewer contacts at these large values. This suggests that during the formation of the packing, some of the kinetic energy is stored as elastic energy in the force chains. If the packing is formed with very little kinetic energy (as in the case of CD-2 and MD-2), fewer large contact forces are present.

\section{CONCLUSIONS}

One conclusion that can be drawn from this work is the importance of sliding contacts. In Sec. IIE we showed that they are associated with the boundary of $\mathbb{F}$, and hence are a sign that the packing is close to yielding. Furthermore, our results suggest that there should be fewer than $2 M-3 N$ sliding contacts (counting nontransmitting contacts as two sliding contacts) in a stable packing, and no counterexamples were found among the 240 configurations that were examined. If a granular packing is slowly loaded, our work predicts that the num- ber of sliding contacts will increase, and reach $2 M-3 N$ when the packing yields. The MD algorithm produces many more sliding contacts than $\mathrm{CD}$. This suggests that packings under CD are much more stable than under MD.

We were able to calculate the local indeterminacy, that is the range of values a given contact force can assume. We found that the contacts with large indeterminacy are also those contacts that make up force chains. Therefore, the primary origin of indeterminacy is that the amplitudes of the force chains can change. This result also suggests that force chains could be understood by investigating the null space of the contact matrix $\mathbf{c}$, since the difference between any two allowed states belongs to this set.

The global indeterminacy measures how much the entire network must be adjusted in order to maximize or minimize a force at a given contact. The reorganizations required for most contacts are significant, even when the local indeterminacy is small. The global indeterminacy can also be used to estimate the diameter of $\mathbb{F}$. This diameter in turn allows one to appreciate the magnitude of changes occurring within the force network. For example, we saw that changing the simulation method changes the force network by an amount roughly equivalent to the diameter of $\mathbb{F}$. Erasing the memory of a CD simulation changes the forces by roughly half as much. Our estimates of the diameter of $\mathbb{F}$ are consistent with those obtained using a Monte Carlo-like procedure to sample $\mathbb{F}$.

Finally, we made several observations about how a packing's "memory" is formed. When a packing is formed violently, with much kinetic energy, some of this energy ends up stored in the contacts. Such packings exhibit stronger force chains and larger contact forces than packings formed gently, with very little kinetic energy. Both $\mathrm{CD}$ and MD simulations show this effect, although it is more significant in the MD simulations.

\section{Acknowledgments}

We thank F. Radjai and J.J. Moreau for help with our contact dynamics program. We acknowledge support from the Deutsche Forschungsgemeinschaft Sondernforschungsbereich 382, Project C15.
[1] H.J. Herrmann and S. Luding, Continuum Mechanics and Thermodynamics 10189 (1998).

[2] C. Bizon, M.D. Shattuck, J.B. Swift, W.D. McCormick, and H.L. Swinney, Phys. Rev. Lett., 8057 (1997).

[3] S. Luding, Phys. Rev. E 63042201 (2001).

[4] B. Bernu and R. Mazighi, J. Phys. A. Math. Gen. 23 5745 (1990).

[5] S.McNamara and W.R.Young, Phys. Fluids A 4496
(1992).

[6] S. Luding and S. McNamara, Granular Matter 1113 (1998).

[7] D. Goldman, M.D. Shattuck, C. Bizon, W.D. McCormick, J.B. Swift, and H.L. Swinney, Phys. Rev. E 574831 (1998).

[8] F.Radjai, L.Brendel, and S. Roux, Phys Rev E 54861 (1996) 
[9] F. Radjai, Ph.D. Thesis, Université de Paris-Sud, 1995.

[10] J.J. Moreau, in Novel Approaches in Civil Engineering, M. Frémond and F. Maceri, eds, Lecture Notes in Applied and Computational Mechanics 141 (2004).

[11] P.A. Cundall and D.L. Strack, Geotechnique 2947 (1979).

[12] J.H. Snoeijer, T.J.H. Vlugt, M. van Hecke, and W. van Saarloos, Phys. Rev. Lett. 92054302 (2004).
[13] J.-N. Roux, Phys. Rev. E 616802 (2000).

[14] T. Unger, J. Kertész, and D.E. Wolf, cond-mat/0403089

[15] T. Elperin and A. Vikhansky, Physica A 260201 (1998).

[16] F. Radjai, M. Jean, J.-J. Moreau, and S. Roux, Phys. Rev. Lett. 77274 (1996).

[17] R. García-Rojo and H. Herrmann, preprint. 